\title{
ORIGINAL ARTICLE The impact of chronic stress on the rat brain lipidome
}

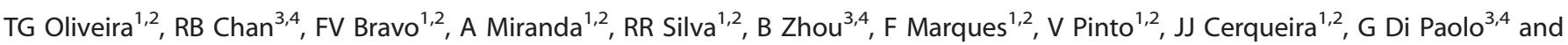 \\ N Sousa ${ }^{1,2}$
}

\begin{abstract}
Chronic stress is a major risk factor for several human disorders that affect modern societies. The brain is a key target of chronic stress. In fact, there is growing evidence indicating that exposure to stress affects learning and memory, decision making and emotional responses, and may even predispose for pathological processes, such as Alzheimer's disease and depression. Lipids are a major constituent of the brain and specifically signaling lipids have been shown to regulate brain function. Here, we used a mass spectrometry-based lipidomic approach to evaluate the impact of a chronic unpredictable stress (CUS) paradigm on the rat brain in a region-specific manner. We found that the prefrontal cortex (PFC) was the area with the highest degree of changes induced by chronic stress. Although the hippocampus presented relevant lipidomic changes, the amygdala and, to a greater extent, the cerebellum presented few lipid changes upon chronic stress exposure. The sphingolipid and phospholipid metabolism were profoundly affected, showing an increase in ceramide (Cer) and a decrease in sphingomyelin (SM) and dihydrosphingomyelin (dhSM) levels, and a decrease in phosphatidylethanolamine (PE) and ether phosphatidylcholine (PCe) and increase in lysophosphatidylethanolamine (LPE) levels, respectively. Furthermore, the fatty-acyl profile of phospholipids and diacylglycerol revealed that chronic stressed rats had higher 38 carbon(38C)-lipid levels in the hippocampus and reduced 36C-lipid levels in the PFC. Finally, lysophosphatidylcholine (LPC) levels in the PFC were found to be correlated with blood corticosterone (CORT) levels. In summary, lipidomic profiling of the effect of chronic stress allowed the identification of dysregulated lipid pathways, revealing putative targets for pharmacological intervention that may potentially be used to modulate stress-induced deficits.
\end{abstract}

Molecular Psychiatry (2016) 21, 80-88; doi:10.1038/mp.2015.14; published online 10 March 2015

\section{INTRODUCTION}

It is well established that prolonged exposure to stress triggers deleterious effects on the brain structure and function. Indeed, animals exposed to chronic stress have functional and morphological impairment in various brain regions, such as the hippocampus, the prefrontal cortex (PFC) and the amygdala. ${ }^{1,2}$ The implications of these stress-induced alterations are multiple, with an impact on learning and memory, ${ }^{3,4}$ decision making ${ }^{5}$ and emotional responses. ${ }^{6}$ Moreover, stress has been linked to pathological processes involved in highly prevalent diseases, such as Alzheimer's disease $^{7}$ and depression. ${ }^{8}$ It is thus crucial to thoroughly understand the molecular basis underlying the pathogenic effects of chronic stress to identify novel targets for therapeutic interventions against stress-induced alterations.

Stress response is characterized by a fast activation of the sympathetic nervous system, followed by a slower activation of the hypothalamic-pituitary-adrenal axis that culminates with the release of glucocorticoids (GCs): cortisol in primates and corticosterone (CORT) in rodents. These hormones, believed to mediate a significant part of the pathologic effects of chronic stress, are derived from cholesterol and typically bind to intracellular receptors. There are two different corticosteroid receptors, the mineralocorticoid receptor and the GC receptor, which dimerize upon ligand binding and translocate to the nucleus, regulating gene transcription; the role of GC receptors in the membrane has been recognized but their functional role is still being scrutinized. ${ }^{9}$ Because CORT has a much higher affinity for mineralocorticoid receptor than for GC receptor, the latter is occupied only in the presence of elevated CORT levels, such as those triggered by chronic stress. ${ }^{10}$ Importantly, it has been shown that chronic unpredictable stress (CUS) leads to major structural and functional hippocampal alterations, ${ }^{4,11,12}$ which are, at least in part, mediated by increased levels of CORT.,11 Therefore, it is critical to gain insights into the molecular mechanisms responsible for the brain manifestations of chronic stress and determine the contribution of CORT-dependent and -independent processes to these manifestations. Growing evidence suggests that stress/GCs may have an impact on brain lipid metabolism. For instance, GCs have been shown to modulate brain lipid signaling via stimulation of phospholipase A2 (PLA2), a key lipid enzyme that cleaves membrane phospholipids into lysophospholipids and arachidonic acid (AA), which are associated with inflammatory processes. ${ }^{13}$ Importantly, it has been shown that chronic stress also alters the expression of a lipid-modifying enzyme, diacylglycerol (DG) lipase $a$, thus modulating the levels of DG and 2 -arachidonoylglycerol in stress-susceptible brain regions. ${ }^{14}$ Moreover, chronic stress was shown to lead to an increase in ceramide (Cer) levels in the mouse hippocampus. ${ }^{15}$

Given the implication of multiple facets of lipid signaling in stress, we reasoned that a comprehensive mass spectrometrybased lipidomic analysis of rodent brain under conditions of stress would be both timely and informative. We evaluated the impact of a 4-week CUS paradigm on various rat brain regions believed to be affected by stress-the hippocampus, the PFC and the amygdala-and as a control we analyzed the cerebellum, which

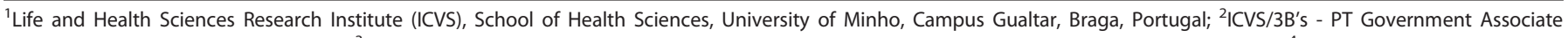

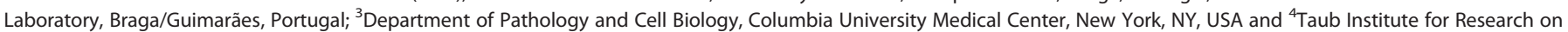

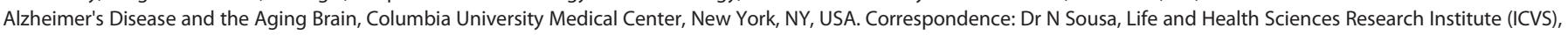
School of Health Sciences, University of Minho, Campus Gualtar, 4710-057 Braga, Portugal.

E-mail: njcsousa@ecsaude.uminho.pt

Received 18 June 2014; revised 28 November 2014; accepted 19 December 2014; published online 10 March 2015 
is potentially a brain region not significantly affected by stress. ${ }^{2}$ Overall, the PFC was the brain area with the highest degree of changes induced by chronic stress. Specifically, sphingolipid metabolism was affected, showing an increase in Cer and a decrease in sphingomyelin (SM) and dihydrosphingomyelin (dhSM) levels. Also, phospholipid metabolism was found to be altered, with decreased phosphatidylethanolamine (PE) and ether phosphatidylcholine (PCe) levels and an increase in lysophosphatidylethanolamine (LPE) level. Furthermore, the fattyacid profile of phospholipids and DG revealed that chronically stressed rats had higher hippocampal 38 carbon (38C)-lipid levels and decreased PFC 36C-lipid levels. Finally, our lipidomic analysis revealed multiple brain lipid species levels to be correlated with blood CORT levels.

\section{MATERIALS AND METHODS}

Animals and treatments

Experiments were conducted in accordance with local regulations (European Union Directive 86/609/EEC) and National Institutes of Health guidelines on animal care and experimentation. Adult ( 2 months old at the beginning of the experiment) male Wistar rats (Charles River Laboratories, Barcelona, Spain) were housed in groups of two under standard laboratory conditions (lights were on from 0800 to 2000 hours; room temperature $22^{\circ} \mathrm{C}$; ad libitum access to food and drink). In our experimental design we tested four different conditions: (1) A group of 10 rats were handled daily and served as controls. (2) A group of 10 rats were submitted to 4 weeks of a CUS paradigm as described by Cerqueira et al..$^{3}$ Briefly, animals were exposed once daily to a stressor $\left(1 \mathrm{~h} \mathrm{day}^{-1}\right)$ of one of several aversive stimuli (cold water $\left(18^{\circ} \mathrm{C}\right)$, vibration, restraint, overcrowding or exposure to a hot air stream); the stressors were presented in random order for the duration of the experiment. (3) A group of 10 animals were used as a vehicle control group, with daily subcutaneous injections of sesame oil for 4 weeks. (4) A group of 10 animals underwent daily subcutaneous injections of synthetic CORT $40 \mathrm{mg} \mathrm{kg}^{-1}$ (Sigma-Aldrich, St. Louis, MO, USA) dissolved in sesame oil for 4 weeks. This CUS paradigm was previously shown to result in persistently elevated plasma levels of CORT, the primary GC of the rat. $^{3}$

To ensure the effectiveness of the experimental procedures, serum CORT levels were measured using a commercially available radioimmunoassay kit (MP Biochemicals, Santa Ana, CA, USA) after the 4-week treatment regimens (between 0800 and 0090 hours). The effectiveness of the CUS paradigm was confirmed with measurement of serum CORT levels, with a $58 \%$ increase in the CUS animals (control group $98.9 \pm 11.8 \mathrm{ng} \mathrm{ml}^{-1}$; CUS group $\left.154.1 \pm 11.8 \mathrm{ng} \mathrm{ml}^{-1} ; P=0.029\right)$, and with behavioral analysis using the elevated plus maze test (see below for methodological details), with a $48.5 \%$ decrease in the fraction of time spent in open arms for CUS animals (control group $38.1 \pm 10.2 \mathrm{~s}$; CUS group $19.6 \pm 11.8 ; P=0.022$ ). One serum sample was lost during the experimental procedure.

\section{Behavioral testing-elevated plus maze}

The elevated plus maze test behavioral paradigm was used to test anxietylike behavior. The elevated plus maze test apparatus was made of black polypropylene (ENV-560; MedAssociates, St. Albans, VT, USA) and consisted of two opposite open arms $(50.8 \times 10.2 \mathrm{~cm})$ and two enclosed arms $(50.8 \times 10.2 \times 40.6 \mathrm{~cm})$ elevated $72.4 \mathrm{~cm}$ above the floor. The junction area between the four arms measured $10 \times 10 \mathrm{~cm}$. A raised edge $(0.5 \mathrm{~cm})$ on the open arms provided additional grip for the rats. Rats were placed individually in the center of the maze facing a closed arm and were allowed $5 \mathrm{~min}$ of free exploration. Behavioral parameters were recorded with the use of an infrared photobeam system connected to a computer with specific software (MedPCIV, MedAssociates) and posteriorly confirmed by video analysis. After each trial, the maze was cleaned with $10 \%$ ethanol. The percentage of time spent in the open arms was taken as an index of anxiety-like behavior. ${ }^{16}$

\section{Annotation of lipid species}

Lipids are annotated with subclass name followed by total fatty-radyl carbons and unsaturation as needed. DG and TG species are annotated similarly, with the addition of fatty-acyl carbon and unsaturation of the product ion. All measured sphingolipids contained d18:1 long-chain base (excepting dhSM species, containing a d18:0 base). All measured acyl-phosphatidylserine (APS) species contained C16:0N-linked fatty-acyl chains.

Analysis of lipids using high-performance liquid chromatography-mass spectrometry

Upon completion of behavioral testing, the animals were killed, macrodissection of the hippocampus, PFC, amygdala and cerebellum was performed, and all tissue samples were immediately collected in tubes, drop-freezed in liquid nitrogen and subsequently stored at $-80^{\circ} \mathrm{C}$ for further processing. Samples from each brain area were processed simultaneously. Tissue lipid extracts were prepared using a modified Bligh/Dyer procedure, spiked with appropriate internal standards, and analyzed using a 6490 Triple Quadrupole LC/MS system (Agilent Technologies, Santa Clara, CA, USA). Glycerophospholipids and sphingolipids were separated with normal-phase HPLC as before, ${ }^{17,18}$ with a few changes. An Agilent Zorbax Rx-Sil column (inner diameter $2.1 \times 100 \mathrm{~mm}$ ) was used under the following conditions: mobile phase A (chloroform:methanol:1 $\mathrm{m}$ ammonium hydroxide, 89.9:10:0.1, v/v) and mobile phase B (chloroform:methanol:water: ammonium hydroxide, 55:39.9:5:0.1, v/v); $95 \%$ A for 2 min, linear gradient to $30 \%$ A over $18 \mathrm{~min}$ and held for $3 \mathrm{~min}$, and linear gradient to $95 \%$ A over $2 \mathrm{~min}$ and held for $6 \mathrm{~min}$. Sterols and glycerolipids were separated with reverse-phase HPLC using an isocratic mobile phase as before ${ }^{17}$ except with an Agilent Zorbax Eclipse XDB-C18 column $(4.6 \times 100 \mathrm{~mm})$. Individual lipid species were measured by multiple reaction monitoring transitions and lipid concentration was calculated by referencing to appropriate internal standards: D5-cholesterol, CE 17:0, 4ME 16:0 diether DG, D5-TG 16:0/18:0/16:0, PA 14:0/14:0, PC 14:0/14:0, PE 14:0/14:0, PG 15:0/15:0, PS 14:0/14:0, LPC 17:0, LPE 14:0, LPI 13:0, BMP 14:0/14:0, SM d18:1/12:0, dhSM d18:0/12:0, Cer d18:1/17:0, GalCer d18:1/12:0, LacCer d18:1/12:0 and Sulf d18:1/17:0 (Avanti Polar Lipids, Alabaster, AL, USA). PI 16:0/16:0 was purchased separately (Echelon Biosciences, Salt Lake City, UT, USA). Some lipid classes did not have commercially available internal standards and hence these lipids were referenced to standards that are closely eluted in the liquid chromatography-mass spectroscopy method: Ether-linked species were normalized to corresponding acyl-linked standards: NAPS to PS 14:0/14:0 and GM3 to PI 16:0/16:0. Lipid concentration was normalized by molar concentration across all species for each sample, and the final data are presented as mean mol\%.

\section{Gene expression measurements by qRT-PCR}

Total RNA was extracted from PFC using the TripleXtractor (Grisp, Porto, Portugal), following the manufacturer's instructions. RNA quality and quantification was assessed in the NanoDrop ND-1000 (NanoDrop, Thermo Scientific, Wilmington, DE, USA) and a total of $1 \mu \mathrm{g}$ of RNA from each sample was reverse transcribed into CDNA using the iScriptTM CDNA Synthesis Kit (Bio-Rad Laboratories, Hercules, CA, USA) following the manufacturer's instructions. Primers used to measure the expression levels of selected mRNA transcripts of Rattus norvegicus and Mus musculus (conserved sequence) by qRT-PCR were designed using Primer3 software, on the basis of the respective GenBank sequences, confirming intron spanning using BLAT software. The reference gene glyceraldehyde 3-phosphate dehydrogenase was used as the internal standard for the normalization of the selected transcript expression. All gene accession numbers and primer sequences are provided in Supplementary Table 1. qRT-PCR was performed on a CFX 96TM real-time system instrument (BioRad Laboratories), with SsoFastTM EvaGreen Supermix (Bio-Rad Laboratories), according to the manufacturer's instructions, using equal amounts of cDNA from each sample. The cycling parameters were one cycle at $95^{\circ} \mathrm{C}$ for $1 \mathrm{~min}$, followed by 39 cycles at $95^{\circ} \mathrm{C}$ for $15 \mathrm{~s}$, annealing temperature (primer specific) for $20 \mathrm{~s}$ and $72{ }^{\circ} \mathrm{C}$ for $20 \mathrm{~s}$, finishing with one cycle at $65^{\circ} \mathrm{C}$ to $95^{\circ} \mathrm{C}$, with an increment of $0.5^{\circ} \mathrm{C}$, for $5 \mathrm{~s}$ (melting curve). Product fluorescence was detected at the end of the elongation cycle. All melting curves exhibited a single sharp peak at the expected temperature.

\section{Data analysis and presentation}

All lipid species and subclasses analyzed were found to have equal variance (data not shown) and were analyzed with an unpaired two-tailed Student $t$-test. CORT levels and elevated plus maze test data were analyzed with an unpaired one-tailed Student $t$-test in all cases; ${ }^{*} P \leqslant 0.05 ;{ }^{* *} P \leqslant 0.01$; ${ }^{* * *} P \leqslant 0.001$. Correlation data analysis was performed using the linear regression model and coefficient of determination and values are expressed as $R^{2}$. Error bars represent the s.e.m. in all cases. 


\section{RESULTS}

Chronic stress affects preferentially the PFC lipidome

In the control group, different rat brain areas exhibited different lipidomic profiles in the absence of treatment. Considering the major lipid species, we observed that free cholesterol is the most abundant lipid in all regions analyzed, presenting higher levels in the cerebellum and proportionally lower levels in the PFC. In contrast, PC, the second most abundant lipid, displayed an opposite pattern with relatively lower levels in the cerebellum and higher levels in the PFC. On a second level of magnitude, SM, PE, plasmalogen PE, PI, PS, Sulf, CE, DG, dhSM and HexCer presented moderately higher levels compared with the remaining minor subclasses evaluated (Figure 1).

Overall, the PFC was the area with the highest degree of changes induced by chronic stress, with sphingolipid metabolism being the most affected (Figure 1 and Supplementary Figure 1). Considering the total lipid species per lipid class, we found that, in the PFC, CUS led to a sphingolipid imbalance with an increase in Cer and lactosylceramide (LacCer) levels and a decrease in SM and
dhSM levels. Other lipid classes were also affected, as observed by a decrease in PE and ether PC (PCe) levels and an increase in LPE and triacylglycerol (TG) levels (Figure 1 and Supplementary Figure 1). A more profound lipid species analysis of the major altered lipid subclasses (Cer, LacCer, SM, dhSM and PE) in the PFC revealed that there was an overall coherent effect on the majority of the analyzed lipid species (Figure 2).

CUS led to a similarly altered sphingolipid pattern in the hippocampus, with an increase in Cer and a decrease in SM levels. Other lipid changes such as an increase in PI levels and decreased APS levels were also observed (Figure 1 and Supplementary Figure 1).

Interestingly, the amygdala presented only minor alterations, with increased levels of 2-hydroxy $\mathrm{N}$-acyl sulfatide [Sulf(2OH)], a myelin lipid, and no significant differences in the cerebellum (Figure 1 and Supplementary Figure 1).

Fatty-acid lipid composition is affected by chronic stress Brain function relies on the homeostasis of cellular membranes, and its perturbation might partially explain the neuronal deficits a
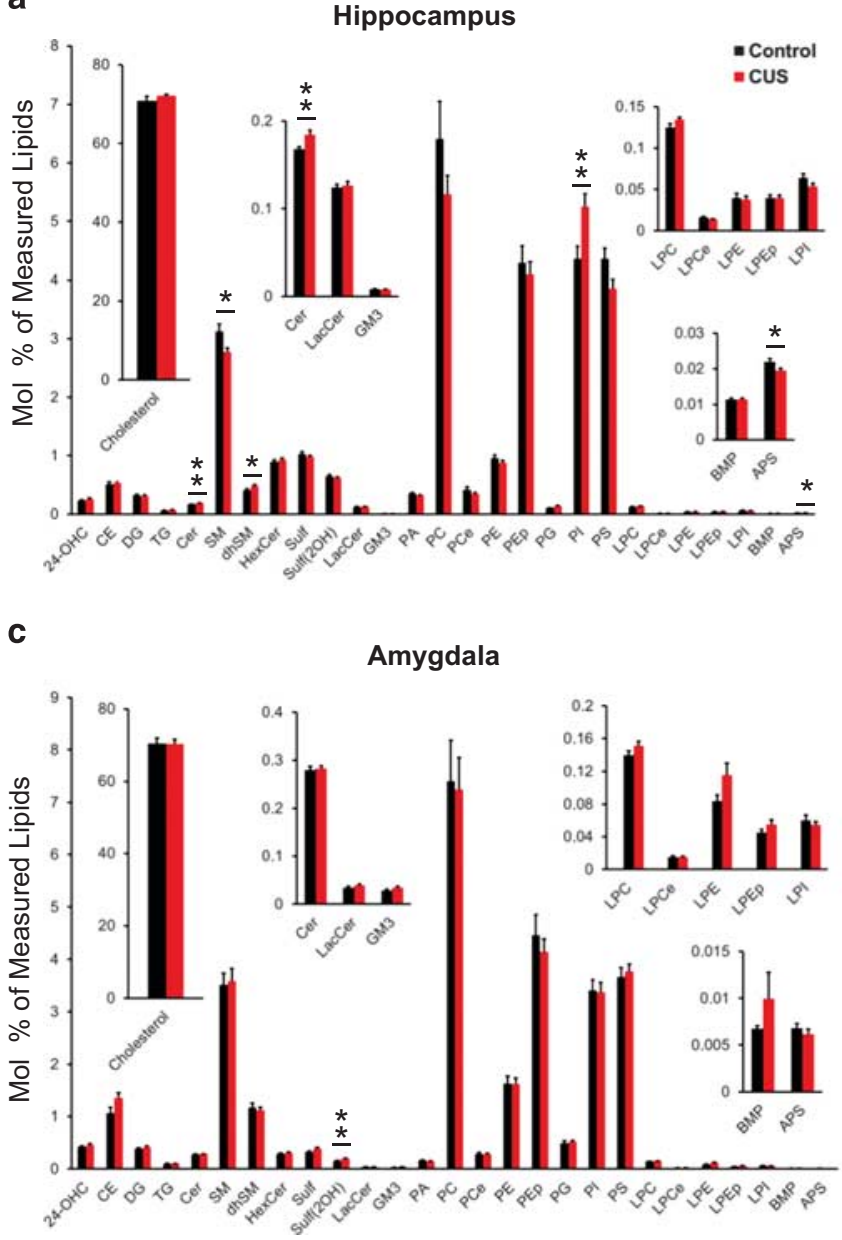

b

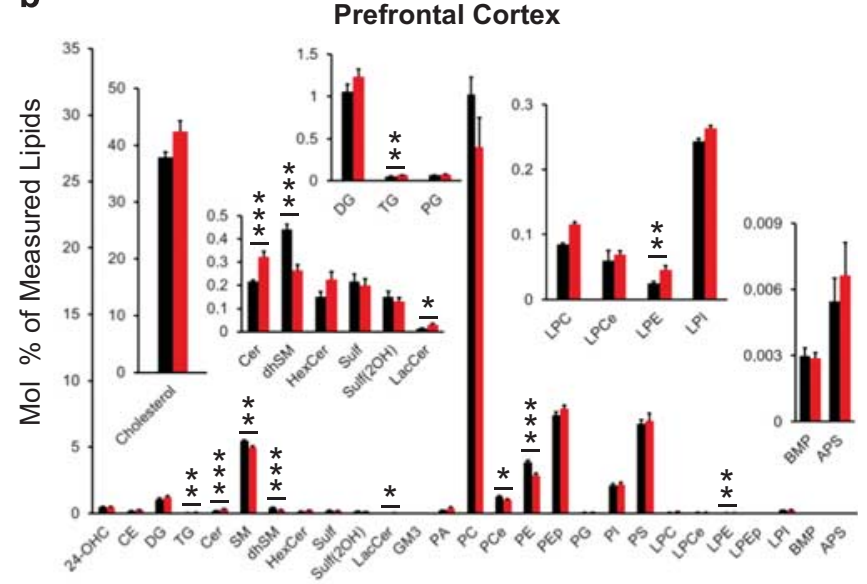

d

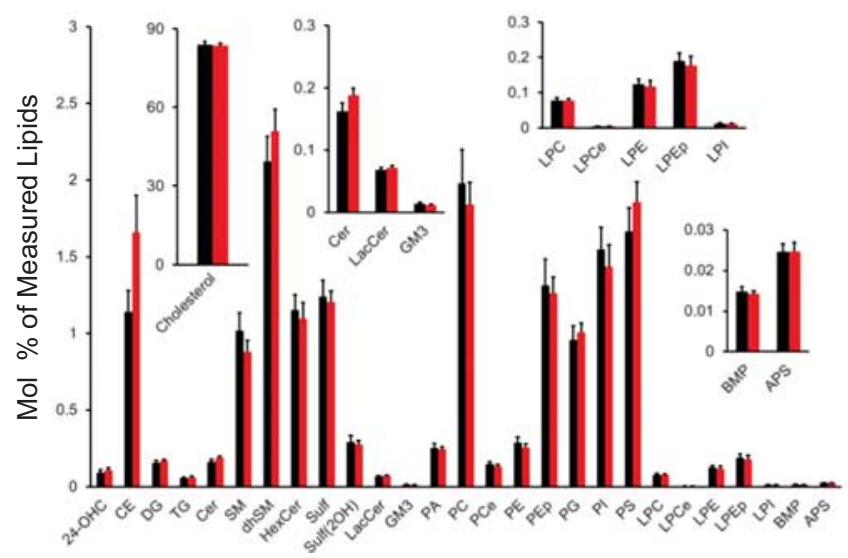

Figure 1. Chronic stress alters the lipid composition in the rat brain. Lipid composition of different areas of the brain of adult rats submitted to 4 weeks of chronic unpredictable stress (CUS, red bars) compared with control rats (black bars). Four brain regions were analyzed: the hippocampus (a), the prefrontal cortex (PFC) (b), the amygdala (c) and the cerebellum (d). Values shown are normalized to measured molar lipid concentration. 24-OHC, 24(S)-hydroxycholesterol; APS, acyl-phosphatidylserine; BMP, bis(monoacylglycero)phosphate; CE, cholesteryl ester; Cer, ceramide; DG, diacylglycerol; dhSM, dihydrosphingomyelin; GM3, monosialodihexosylganglioside; HexCer, hexosylceramide; LacCer, lactosylceramide; LPC, lysophosphatidylcholine; LPCe, ether lysophosphatidylcholine; LPE, lysophosphatidylethanolamine; LPEp, plasmalogen LPE; LPI, lysophosphatidylinositol; PA, phosphatidic acid; PC, phosphatidylcholine; PCe, ether phosphatidylcholine; PE, phosphatidylethanolamine; PEp, plasmalogen PE; PG, phosphatidylglycerol; PI, phosphatidylinositol; PS, phosphatidylserine; SM, sphingomyelin; Sulf, sulfatides; Sulf $(2 \mathrm{OH})$, 2- hydroxy $N$-acyl sulfatide; TG, triacylglycerol. $N=10$ per group Results are presented as mean and bars as s.e.m. ${ }^{*} P \leqslant 0.05$; ${ }^{* *} P \leqslant 0.01 ;{ }^{* * *} P \leqslant 0.001$ by the unpaired two-tailed Student $t$-test. 
a
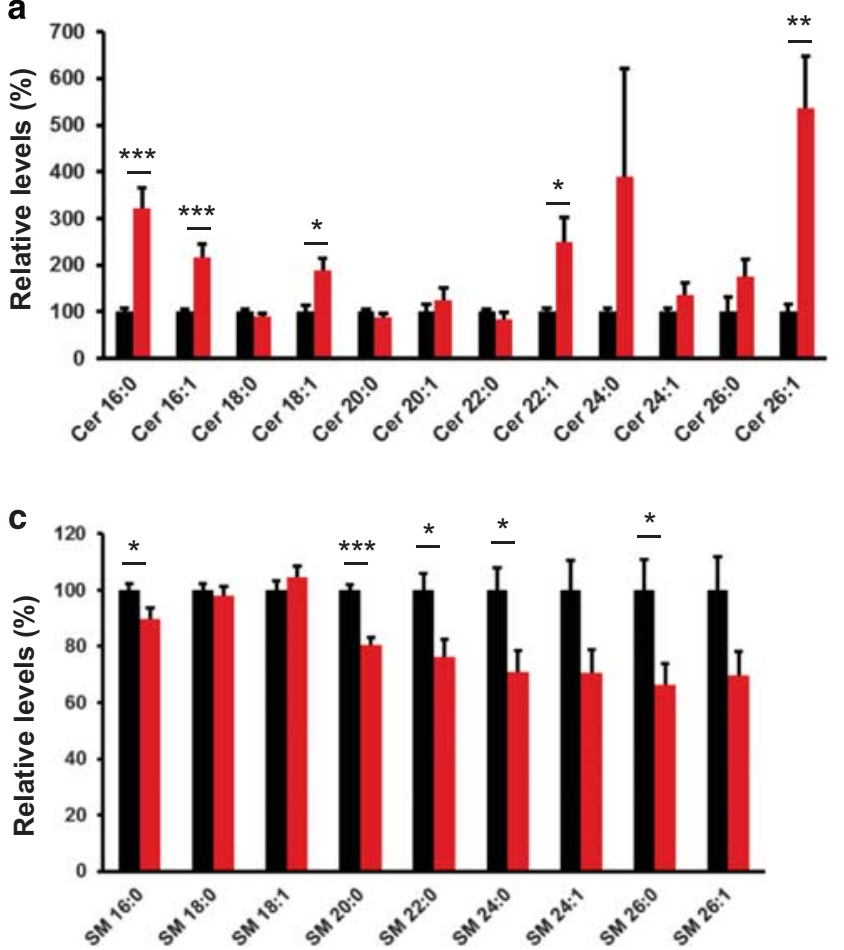

b

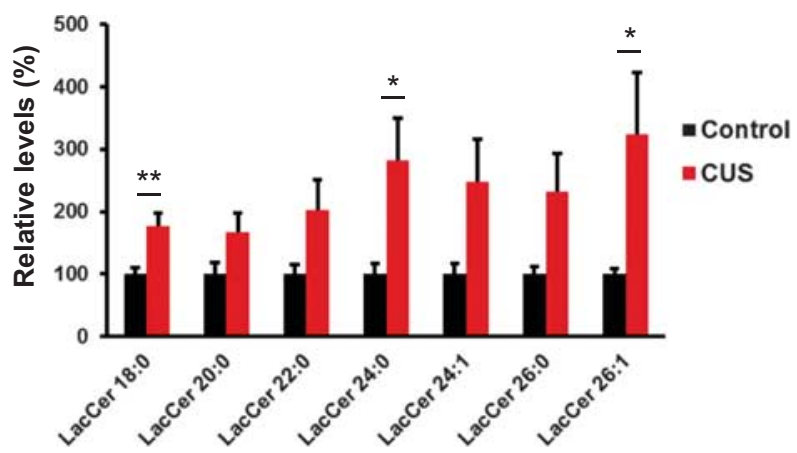

d

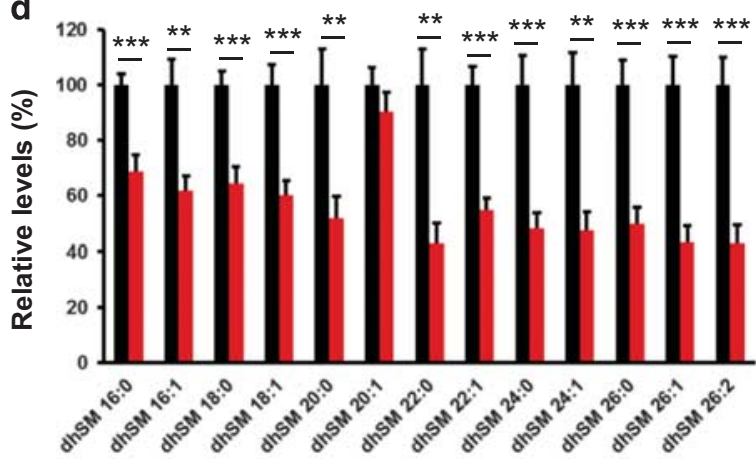

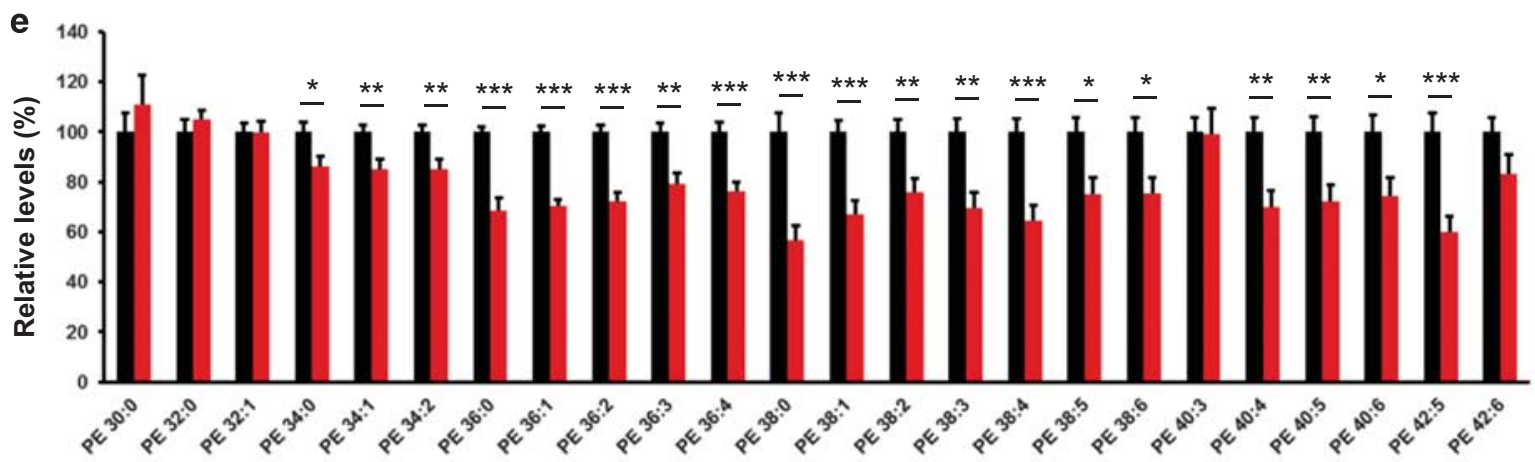

Figure 2. Chronic stress has a significant impact on sphingolipid and PE metabolism in the prefrontal cortex (PFC). PFC lipid species composition of adult rats subjected to 4 weeks of chronic unpredictable stress (CUS, red bars) compared with control rats (black bars) submitted to handling. Analysis showed increases in several Cer (a) and all lactosylceramide (b) species and decreases in many SM (c), dhSM (d) and PE (e) species. y values expressed as relative levels to control animals. Cer, ceramide; dhSM, dihydrosphingomyelin; LacCer, lactosylceramide; $\mathrm{PE}$, phosphatidylethanolamine; $\mathrm{SM}$, sphingomyelin. $N=10$ per group. Results are presented as mean and bars as standard error of the mean. ${ }^{*} P \leqslant 0.05 ;{ }^{* *} P \leqslant 0.01 ;{ }^{* * *} P \leqslant 0.001$.

observed in various neurological conditions. Both the carbon chain length and the degree of saturation of the fatty-acyls that are part of membrane lipids determine membrane biophysical properties. Therefore, the modulation of the composition of its fatty-acyl chains can potentially affect neuronal functioning, at least in part through altered function of membrane-bound proteins. $^{19,20}$

In our study we performed another level of analysis focusing on the fatty-acyl chain profile. Our data show that CUS led to a significant alteration in the fatty-acyl chain profile of phospholipids and DG, with increased levels of long-chain fatty-acyls with $38 \mathrm{C}$ and an increase in polyunsaturated fatty-acyls with four double bonds in the hippocampus (Figure 3). Whereas in the PFC we observed a decrease in $36 \mathrm{C}$ fatty-acyls, in the cerebellum we observed an increase in its levels. Interestingly, with regard to the effects of CUS in sphingolipid composition, only the PFC was affected with an increase in short-chain fatty-acyls (16C) and a decrease in medium-length (20C) fatty-acyls (Figure 3).

Specific lipid species are correlated with blood CORT levels after chronic stress exposure

The stress response is characterized by an acute activation of the sympathetic nervous system, followed by a slower activation of the hypothalamic-pituitary-adrenal axis that culminates with the release of GCs, such as cortisol in primates and CORT in rodents. To test which lipid species were associated with serum CORT levels, we performed a full-scale unbiased correlation analysis between serum levels of this hormone and abundance of the 359 quantifiable lipid species in each brain region. We defined a cutoff for the correlation index of $0,4\left(R^{2}\right)$ in order to limit the hits in our analysis (Figure 4a). We found that CORT serum levels were 

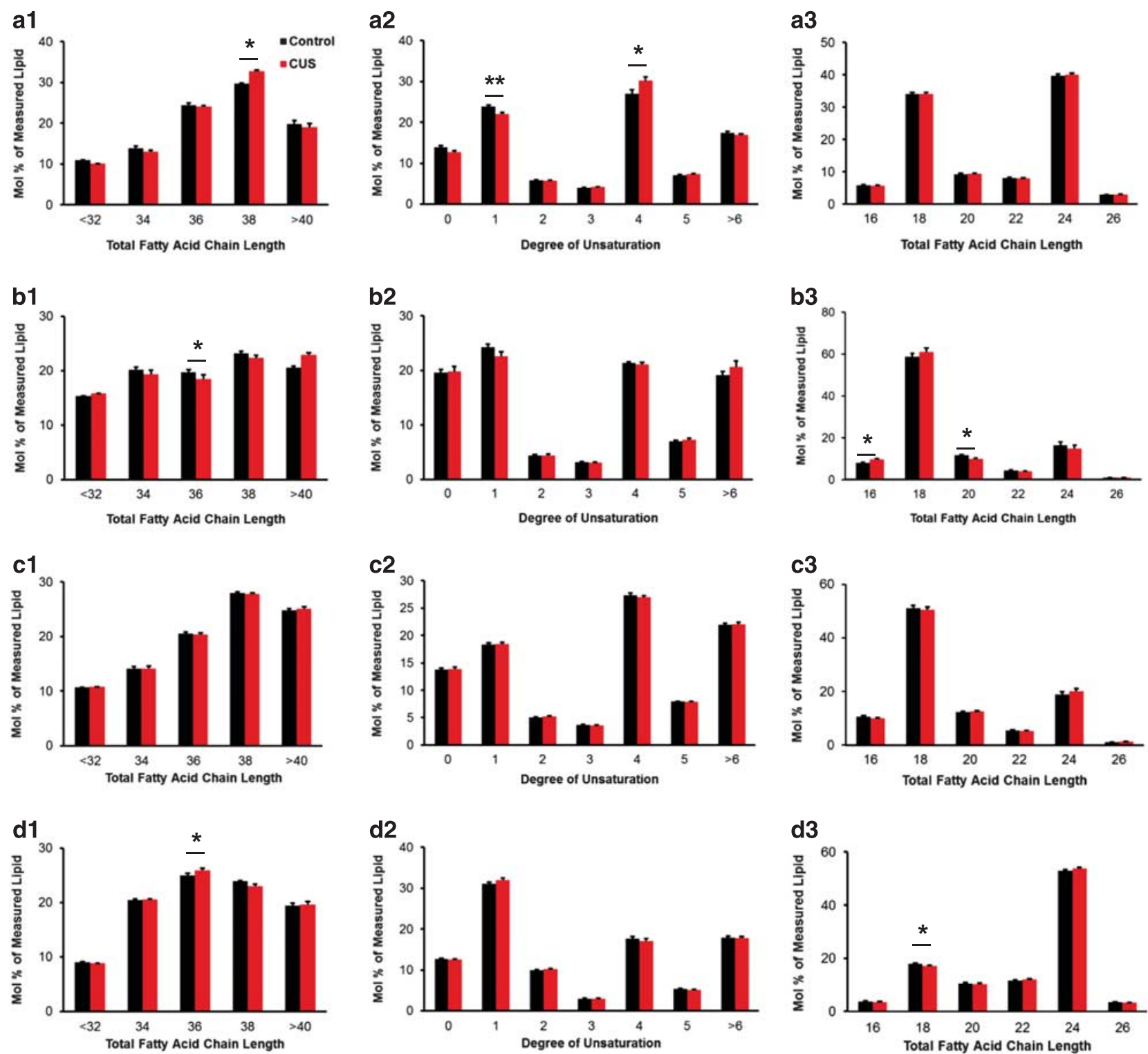

Figure 3. Altered fatty-acid composition after chronic unpredictable stress exposure (CUS) in different rat brain regions. Analysis of fatty-acyl composition of diacyl-glycerophospholipids and DG species by total carbon chain length (a1, b1, c1, d1), total degree of unsaturation (a2, b2, c2, d2) and sphingolipid $\mathrm{N}$-acyl chain lengths (a3, b3, c3, d3). Values are expressed as mol\%+s.e.m of included lipid species. Lipid tissue extracts from the hippocampus (a1-a3), prefrontal cortex (PFC) (b1-b3), amygdala (c1-c3) and cerebellum (d1-d3) were analyzed. Adult rats subjected to 4 weeks of CUS (red bars) compared with control rats (black bars) submitted to handling. $N=10$ per group. ${ }^{*} P \leqslant 0.05 ;{ }^{*} P \leqslant 0.01$; $* * * P \leqslant 0.001$.

directly correlated with PFC levels of total levels of lysophosphatidylcholine (LPC) (Figure 4b1) and inversely correlated with total SM levels (Figure 4b2). Moreover, various LPC species in PFC, LPC 20:4 (Figure 4b3), LPC 20:3 (data not shown), LPC 16:0 (data not shown) and PA 40:5 (Figure 4b4) levels were found to be directly correlated with serum CORT levels. In the hippocampus we found a single correlation hit for LPC 20:4 (Figure 4c). In the amygdala LPC 20:4 (Figure 4e) was also found to be correlated with blood CORT levels, as well as phosphatidylserine (PS) 38:1 levels (data not shown). Finally, no correlation hits were found in the cerebellum.

Common lipid changes after chronic stress and exogenous CORT exposure

In order to gain further insight into which lipid species alterations induced by CUS are in fact mediated by CORT, we evaluated the impact of exogenous administration of CORT in the PFC lipid profile and the lipid-induced alterations common to both the CUS group and the CORT-injected animals. We found a common increase in Cer 18:1, PG 36:1, phosphatidic acid (PA) 40:5, PA 40:6, LPC 16:0 and a common decrease in multiple PC, PCe and PE lipid species (Figure 5).

Stress-induced lipid changes in the PFC are independent of changes in enzymatic expression levels

In light of our comprehensive lipid analysis, and in accordance with previous studies showing altered transcriptomic levels in the PFC in a mouse model of chronic stress, ${ }^{21}$ one possibility was that the lipid changes we observed in the PFC after exposure to CUS were due to expression level alterations of enzymatic lipid modulators. We measured mRNA levels of some of our most likely candidates, namely, Smpd1—acidic sphingomyelinase, 


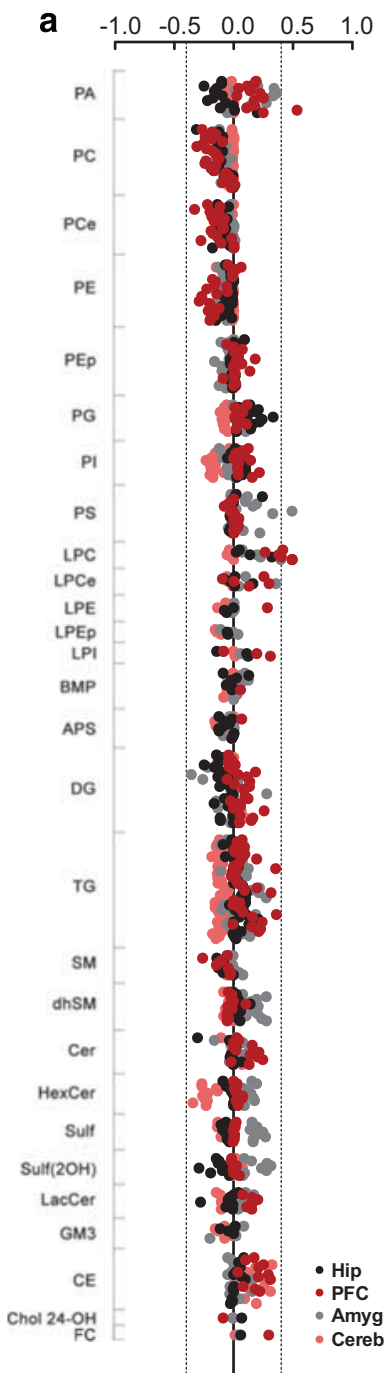

b1

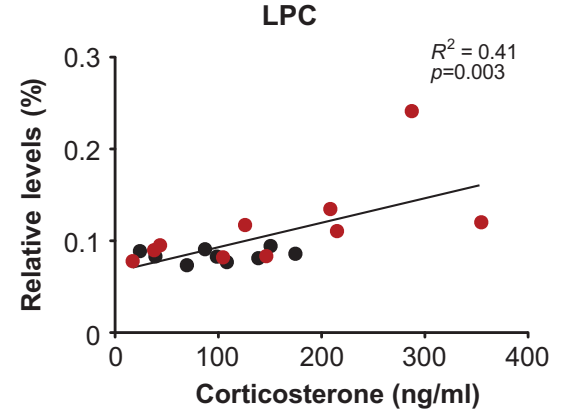

b3

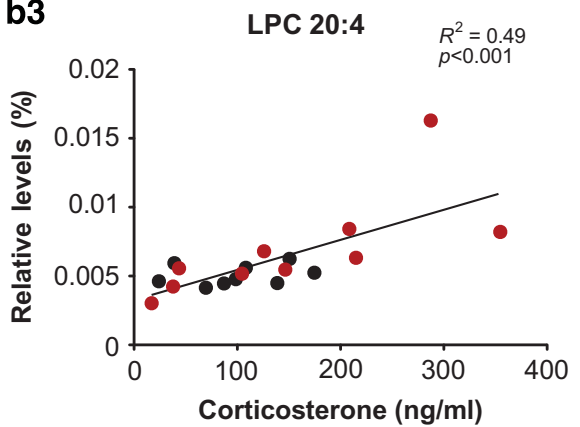

c

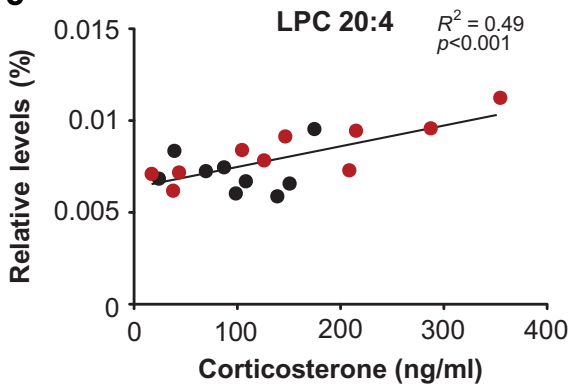

b2

SM

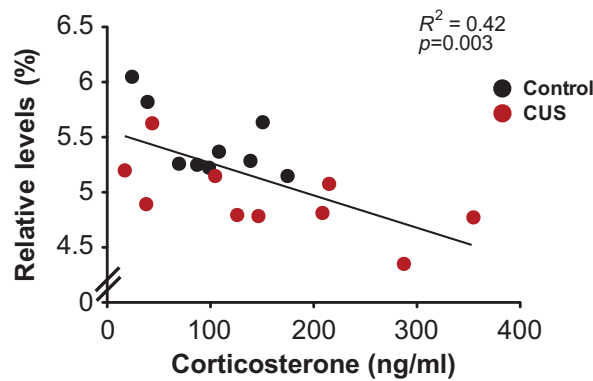

b4
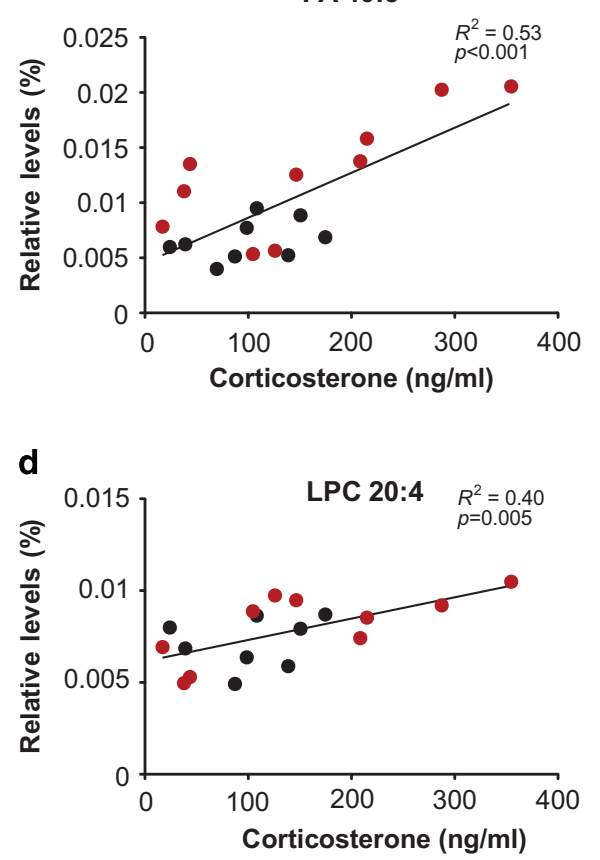

Figure 4. Specific lipid species brain levels are directly correlated with blood corticosterone (CORT) levels. (a) Correlation levels between all lipid species analyzed and blood CORT levels. Lipid species are grouped per lipid class, and rat brain areas are represented with different colored dots: hippocampus (Hip—black), prefrontal cortex (PFC—red), amygdala (Amyg-gray) and cerebellum (Cereb—pink). $R^{2}$ levels are represented from $(-1)$ to $(+1)$, with positive values indicating a positive correlation and negative values indicating a negative correlation. (b-e) For each brain area analyzed, PFC (b), hippocampus (c) and amygdala (d), the major positive lipid species CORT-correlation hits are represented. Not all positive hits are represented in the figure. Adult rats submitted to 4 weeks of chronic unpredictable stress (CUS, red dots) and handled controls (black dots) are represented. (b1-4) Selected lipid species with a higher degree of correlation index in the PFC. (c1) Selected lipid species with a higher degree of correlation index in the hippocampus. (d) Selected lipid species with a higher degree of correlation index in the amygdala. 24-OHC, 24(S)-hydroxycholesterol; APS, acyl-phosphatidylserine; BMP, bis(monoacylglycero)phosphate; CE, cholesteryl ester; Cer, ceramide; DG, diacylglycerol; dhSM, dihydrosphingomyelin; GM3, monosialodihexosylganglioside; HexCer, hexosylceramide; LacCer, lactosylceramide; LPC, lysophosphatidylcholine; LPCe, ether lysophosphatidylcholine; LPE, lysophosphatidylethanolamine; LPEp, plasmalogen LPE; LPI, lysophosphatidylinositol; PA, phosphatidic acid; PC, phosphatidylcholine; PCe, ether PC; PE, phosphatidylethanolamine; PEp, plasmalogen PE; PG, phosphatidylglycerol; PI, phosphatidylinositol; PS, phosphatidylserine; SM, sphingomyelin; Sulf, sulfatides; Sulf(2OH), 2-hydroxy $N$-acyl sulfatide; TG, triacylglycerol. $N=10$ per group.

Smpd2-neutral sphingomyelinase, Pla2g2C, Pla2G4a, Pla2g5, Pla2g6, PId1 and Pld2 (Supplementary Figure 2). We found no differences in the expression levels of our candidate enzymatic hits, suggesting that at least some of the lipid changes we found are independent of expression level regulation of the main catalytical enzymes of our candidate lipid pathways.

\section{DISCUSSION/CONCLUSIONS}

In the present study we performed a detailed lipidomic analysis to understand the impact of chronic stress in distinct brain areas. So far, very few studies have analyzed the impact of either acute ${ }^{22}$ or chronic stress ${ }^{23,24}$ in lipid signaling using a lipidomic approach, the focus has been predominantly on the role of endocannabinoids as modulators of stress-associated pathways. ${ }^{25}$

This thorough analysis of the rodent brain lipidome unmasks the PFC as a key target of stress, with the highest degree of lipid changes induced by chronic stress. Specifically, in the PFC, sphingolipid metabolism was highly affected with increased Cer and decreased SM and dhSM levels, and phospholipid metabolism with decreased PE and increased LPE levels. This region vulnerability fits with previous studies showing that the PFC is the brain 


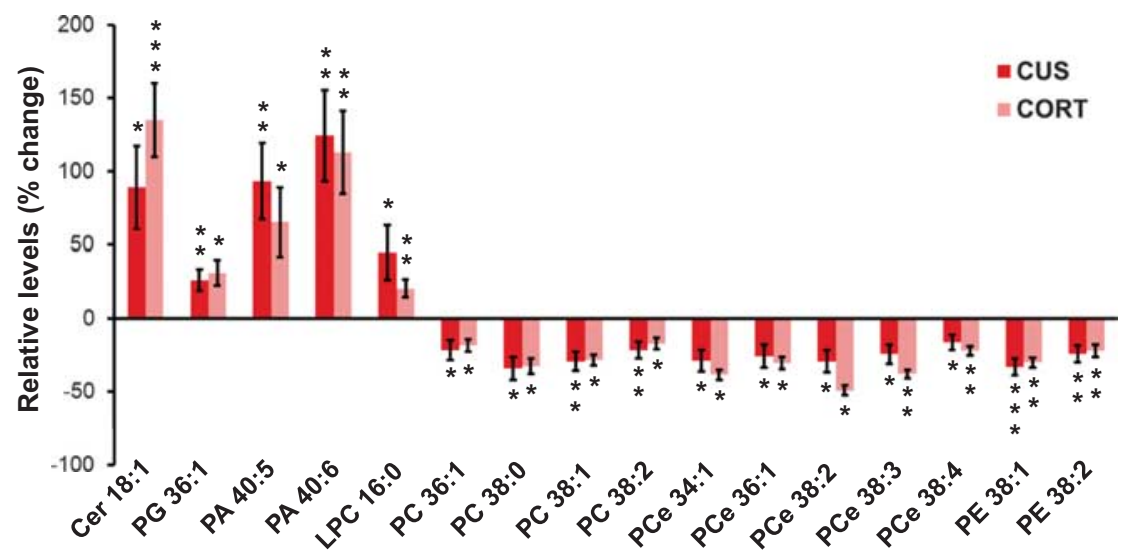

Figure 5. Common prefrontal cortex (PFC) lipid changes after chronic unpredictable stress (CUS) and exogenous corticosterone (CORT) exposure. Common altered lipid species composition in the PFC of adult rats submitted to 4 weeks of CUS or (red bars) to 4 weeks of subcutaneous CORT ( $40 \mathrm{mg} \mathrm{kg}^{-1}$ ) injections (pink bars). Results are expressed as percentual change ratio with each control group; for the CUS the ratio values are relative to control handled animals; for the CORT-injected animals the ratio values are relative to vehicle-injected animals. Cer, ceramide; LPC, lysophosphatidylcholine; PA, phosphatidic acid; PC, phosphatidylcholine; PCe, ether PC; PE, phosphatidylethanolamine; PEp, plasmalogen PE; PG, phosphatidylglycerol. $N=10$ per group. Results are presented as mean and bars as s.e.m. ${ }^{*} P \leqslant 0.05 ;{ }^{* *} P \leqslant 0.01$; $* * * P \leqslant 0.001$.

region displaying major lipid alterations after the use of maprotiline, an antidepressant. ${ }^{26}$ In line with the PFC changes, we found that the hippocampus lipid profile also displayed sphingolipid metabolic changes and increased PI levels. The similar pattern of lipid changes is interesting, as previous studies demonstrated the PFC and the hippocampus to be functionally and structurally impaired after chronic stress exposure. ${ }^{1,2}$ The lipid alterations found in our study suggest that sphingolipid modulating enzymes may be dysregulated in both regions, either due to increased sphingomyelinase (SMase) activity (which catalyzes the hydrolysis of SM and dhSM into Cer and dhCer, respectively) or decreased SM synthase activity (which catalyzes the opposite reaction). ${ }^{27}$ Alternatively, other pathways for Cer formation, such as de novo synthesis or hydrolysis of more complex sphingolipids, cannot be excluded as potential contributors to the observed increase in Cer levels. ${ }^{28}$ This is in accordance with what was shown in other studies, in which hippocampal levels of Cer were found to be increased in a CUS mouse model. ${ }^{15}$ Importantly, these sphingolipid alterations can potentially alter the organization of specific lipid microdomains, such as lipid rafts, with implications for brain function. $^{29}$

The combination of decreased PE and increased LPE levels suggests a hyperactive state of a PLA2 isoform ${ }^{30}$ in the PFC after CUS exposure. Interestingly, decreased levels and pharmacologic inhibition of acidic SMase prevented CUS-induced alterations, ${ }^{15}$ and the downregulation of calcium-independent PLA2 (iPLA2) blocked both the behavior-induced alterations and the PFC lipid profile owing to maprotiline chronic treatment. ${ }^{31}$ Also noticeable is the observation that PLA2 activation is associated with increased inflammation. In a genetically induced model of neurodegeneration, it was shown that specific lysophospholipids were responsible for microglial activation and that cytosolic PLA2 (CPLA2) inhibition protected neurons from associated local inflammation and glutamate-mediated neurotoxicity. ${ }^{32}$ Besides comparing the individual species we also analyzed the fatty-acyl chain profile of both phospholipids/DG and sphingolipids. In summary, we found that CUS led to a decrease in 36C fatty-acyl phospholipids/DG levels in the PFC and to an increase in $38 \mathrm{C}$ and four double-bond polyunsaturated phospholipids/DG levels in the hippocampus. Interestingly, the hippocampal data indicate the possible accumulation of phospholipids containing AA (20:4) as part of the fattyacyl composition. This is supported by findings in a depressive-like rat model in which a similar pattern of increased levels of
AA-containing phospholipids and decreased docosahexaenoic acid (DHA)-containing phospholipids occurred in the PFC. ${ }^{33,34}$ Also, diets rich in polyunsaturated fatty acids (PUFA), such as fish oil diet, showed an increase in DHA-containing phospholipids PC 40:6 and PC 38:6 and a decrease in AA-containing phospholipids PC $38: 4$ and PC $36: 4{ }^{35}$ The DHA-treated rats had decreased levels of AA-containing phospholipids with concomitant prevention of the lipid alterations induced by aging. ${ }^{36}$ Finally, diets deficient in n-3 PUFA led to decreased DHA levels and increased DHA half-life due to a downregulation of iPLA2 levels and upregulation of both secreted PLA2 (sPLA2) and CPLA2 in the frontal cortex. ${ }^{37}$ Overall, our data, and previous studies, suggest that $A A$ accumulation in the hippocampus might be deleterious, whereas DHA accumulation in the frontal cortex might be protective in the context of neurodegeneration. As it was previously proposed that CPLA2 preferentially releases $A A$ and that iPLA2 preferentially cleaves DHA-containing phospholipids, ${ }^{38}$ it is plausible that there is a dysregulation of iPLA2 in the PFC and of CPLA2 in the hippocampus (as discussed $i^{37}$ and $^{38}$ ). Moreover, patients with depression were found to have increased ratio blood levels of $\mathrm{n}-6 / \mathrm{n}-3$ PUFA, ${ }^{39}$ and chronic stress in rodents altered lipoprotein blood and liver metabolism. ${ }^{40,41}$ Of note, circulating FA can reach the brain and be incorporated in the cells of the central nervous system. ${ }^{42}$ Therefore, all these aspects are of critical relevance for our understanding of the lipid metabolism crosstalk between the central nervous system and the periphery in physiological and pathological conditions, and should be addressed in future studies. As elevations in CORT levels are a critical element in the stress response, we tested which of the stress effects in brain lipidomics could be attributed to hypercortisolemia. First, with an unbiased strategy, we identified which lipid species had a higher degree of correlation with endogenous CORT levels; second, we identified the common alterations in the PFC lipid profile of both CUS- and CORT-injected animals. In the PFC we found that CORT serum levels were directly correlated with multiple LPC species levels, and inversely correlated with total SM levels. Interestingly, we found that, specifically, LPC 20:4 was found as a positive correlation hit in all brain areas studied except the cerebellum. This is in accordance with the also observed accumulation of AA-containing phospholipids, which is thought to have a deleterious effect. Among the few studies addressing the role of LPC 20:4, it was shown that it can promote inflammatory events through the donation of $A A$, which is proposed to be released in a 
CPLA2-dependent way. ${ }^{43}$ Moreover, both CUS- and CORT-injected animals had increased levels of Cer 18:1 and LPC 16:0 and decreased levels in multiple PC, PCe and PE species, suggesting that CORT might be leading to an activation of PLA2 and SMase in the PFC of stressed animals. Interestingly, PA 40:5 PFC levels were directly correlated with endogenous blood CORT levels, and PA 40:5 and PA 40:6 levels were both increased in the PFC of CUSand CORT-injected animals, which suggests that phospholipase D (PLD)-dependent pathways might also be activated by GCs. This is in accordance with previous reports showing increased PLD activity after treatment with a synthetic corticosteroid. ${ }^{44}$ The latter is likely to result from the GC-induced calcium influx, ${ }^{45}$ a known activator of PLD. ${ }^{46}$ Interestingly, PLD activation was also shown to be detrimental in other neurodegeneration conditions. ${ }^{47,48}$

In summary, herein we identified the lipid alterations induced by chronic stress and CORT in target brain areas. Our data show that the metabolism of sphingolipids and phospholipids is significantly altered by stress in both the PFC and the hippocampus, suggesting SMase, PLA2 and PLD as potential therapeutical targets for stress-related disorders. These alterations in lipid signaling pathways have several implications in physiological mechanisms, such as neurogenesis and synaptic plasticity, known to be implicated in several stress-related disorders, such as emotional disorders, ${ }^{15,49-51}$ and eventually in other psychiatric disorders, such as schizophrenia. ${ }^{51-54}$ Given that there are different SMase, PLA2 and PLD isozymes, future lipidomic studies addressing the impact of lipid signaling-targeted therapeutical drugs will provide pharmacological mechanistic insights into choosing the best candidate drug in a given pathological condition. Also, other nonpharmacological approaches, such as lifestyle changes and therapeutic diets with previously predetermined brain lipid profiles, may prove of interest in these conditions.

\section{CONFLICT OF INTEREST}

The authors declare no conflict of interest.

\section{ACKNOWLEDGMENTS}

Funding by Fundação para a Ciência e Tecnologia (PTDC/SAU-NMC/118971/2010) and by the North Region Operational Program (ON.2-O Novo Norte), under Quadro de Referência Estratégico Nacional (QREN) and through Fundo Europeu de Desenvolvimento Regional (FEDER). GDP is funded by NIH grants R01 NS056049 and P50 AG008702 (to Scott Small).

\section{REFERENCES}

1 Lupien SJ, McEwen BS, Gunnar MR, Heim C. Effects of stress throughout the lifespan on the brain, behaviour and cognition. Nat Rev Neurosci 2009; 10: 434-445.

2 Sousa N, Almeida OF. Disconnection and reconnection: the morphological basis of (mal)adaptation to stress. Trends Neurosci 2012; 35: 742-751.

3 Cerqueira JJ, Mailliet F, Almeida OF, Jay TM, Sousa N. The prefrontal cortex as a key target of the maladaptive response to stress. J Neurosci 2007; 27: 2781-2787.

4 Pinto V, Costa JC, Morgado P, Mota C, Miranda A, Bravo FV et al. Differential impact of chronic stress along the hippocampal dorsal-ventral axis. Brain Struct Func 2014. (e-pub ahead of print), 6 February 2014.

5 Dias-Ferreira E, Sousa JC, Melo I, Morgado P, Mesquita AR, Cerqueira JJ et al. Chronic stress causes frontostriatal reorganization and affects decision-making. Science 2009; 325: 621-625.

6 Roozendaal B, McEwen BS, Chattarji S. Stress memory and the amygdala. Nat Rev Neurosci 2009; 10: 423-433.

7 Catania C, Sotiropoulos I, Silva R, Onofri C, Breen KC, Sousa N et al. The amyloidogenic potential and behavioral correlates of stress. Mol Psychiatry 2009; 14: 95-105.

8 Bessa JM, Ferreira D, Melo I, Marques F, Cerqueira JJ, Palha JA et al. The moodimproving actions of antidepressants do not depend on neurogenesis but are associated with neuronal remodeling. Mol Psychiatry 2009; 148: 764-773, 739.

9 Sousa N, Almeida OFX. Disconnection and reconnection: the morphological basis of (mal) adaptation to stress. Trends Neurosci 2012; 35: 742-751.
10 Sousa N, Cerqueira JJ, Almeida OFX. Corticosteroid receptors and neuroplasticity. Brain Res Rev 2008; 57: 561-570.

11 Sousa N, Lukoyanov NV, Madeira MD, Almeida OFX, Paula-Barbosa MM. Reorganization of the morphology of hippocampal neurites and synapses after stressinduced damage correlates with behavioral improvement. Neuroscience 2000; 97: 253-266.

12 Sousa N, Almeida OFX. Corticosteroids: sculptors of the hippocampal formation. Rev Neurosci 2002; 13: 59-84.

13 Malcher-Lopes R, Franco A, Tasker JG. Glucocorticoids shift arachidonic acid metabolism toward endocannabinoid synthesis: a non-genomic antiinflammatory switch. Eur J Pharmacol 2008; 583: 322-339.

14 Patel S, Kingsley PJ, Mackie K, Marnett L, Winder DG. Repeated homotypic stress elevates 2-arachidonoylglycerol levels and enhances short-term endocannabinoid signaling at inhibitory synapses in basolateral amygdala. Neuropsychopharmacology 2009; 34: 2699-2709.

15 Gulbins E, Palmada M, Reichel M, Luth A, Bohmer C, Amato D et al. Acid sphingomyelinase-ceramide system mediates effects of antidepressant drugs. Nat Med 2013; 19: 934-938.

16 Bessa JM, Mesquita AR, Oliveira M, Pego JM, Cerqueira JJ, Palha JA et al. A transdimensional approach to the behavioral aspects of depression. Front Behav Neurosci 2009; 3: 1.

17 Chan RB, Oliveira TG, Cortes EP, Honig LS, Duff KE, Small SA et al. Comparative lipidomic analysis of mouse and human brain with alzheimer disease. J Biol Chem 2012; 287: 2678-2688.

18 Dumont M, Stack C, Elipenahli C, Jainuddin S, Gerges M, Starkova N et al. Bezafibrate administration improves behavioral deficits and tau pathology in P301S mice. Hum Mol Genet 2012; 21: 5091-5105.

19 Wenk MR. Lipidomics: new tools and applications. Cell 2010; 143: 888-895.

20 Carta M, Lanore F, Rebola N, Szabo Z, Da Silva SV, Lourenco J et al. Membrane lipids tune synaptic transmission by direct modulation of presynaptic potassium channels. Neuron 2014; 81: 787-799.

21 Lisowski P, Wieczorek M, Goscik J, Juszczak GR, Stankiewicz AM, Zwierzchowski L et al. Effects of Chronic Stress on Prefrontal Cortex Transcriptome in Mice Displaying Different Genetic Backgrounds. J Mol Neurosci 2013; 50: 33-57.

22 Gregg LC, Jung KM, Spradley JM, Nyilas R, Suplita RL 2nd, Zimmer A et al. Activation of type 5 metabotropic glutamate receptors and diacylglycerol lipasealpha initiates 2- arachidonoylglycerol formation and endocannabinoid-mediated analgesia. J Neurosci 2012; 32: 9457-9468.

23 Faria R, Santana MM, Aveleira CA, Simoes C, Maciel E, Melo T et al. Alterations in phospholipidomic profile in the brain of mouse model of depression induced by chronic unpredictable stress. Neuroscience 2014; 273: 1-11.

24 Hill MN, Patel S, Carrier EJ, Rademacher DJ, Ormerod BK, Hillard CJ et al. Downregulation of endocannabinoid signaling in the hippocampus following chronic unpredictable stress. Neuropsychopharmacology 2005; 30: 508-515.

25 Hillard CJ. Stress regulates endocannabinoid-CB1 receptor signaling. Semin Immunol 2014; 26: 380-388.

26 Lee LH, Shui G, Farooqui AA, Wenk MR, Tan CH, Ong WY. Lipidomic analyses of the mouse brain after antidepressant treatment: evidence for endogenous release of long- chain fatty acids? Int J Neuropsychopharmacol 2009; 12: 953-964.

27 Gault CR, Obeid LM, Hannun YA. An overview of sphingolipid metabolism: from synthesis to breakdown. Adv Exp Med and Biol 2010; 688: 1-23.

28 Kornhuber J, Muller CP, Becker KA, Reichel M, Gulbins E. The ceramide system as a novel antidepressant target. Trends Pharmacol Sci 2014; 35: 293-304.

29 Simons K, Gerl MJ. Revitalizing membrane rafts: new tools and insights. Nat Rev Mol Cell Biol 2010; 11: 688-699.

30 Dennis EA, Cao J, Hsu YH, Magrioti V, Kokotos G. Phospholipase A2 enzymes: physical structure, biological function, disease implication, chemical inhibition, and therapeutic intervention. Chem Rev 2011; 111: 6130-6185.

31 Lee LH, Tan CH, Shui G, Wenk MR, Ong WY. Role of prefrontal cortical calcium independent phospholipase $\mathrm{A}(2)$ in antidepressant-like effect of maprotiline. Int J Neuropsychopharmacol 2012; 15: 1087-1098.

32 Sundaram JR, Chan ES, Poore CP, Pareek TK, Cheong WF, Shui G et al. Cdk5/p25induced cytosolic PLA2-mediated lysophosphatidylcholine production regulates neuroinflammation and triggers neurodegeneration. J Neurosci 2012; 32: 1020-1034.

33 Green P, Gispan-Herman I, Yadid G. Increased arachidonic acid concentration in the brain of Flinders Sensitive Line rats, an animal model of depression. $J$ Lipid Res 2005; 46: 1093-1096.

34 Green P, Anyakoha N, Yadid G, Gispan-Herman I, Nicolaou A. Arachidonic acidcontaining phosphatidylcholine species are increased in selected brain regions of a depressive animal model: implications for pathophysiology. Prostaglandins Leukot Essent Fatty Acids 2009; 80: 213-220.

35 Lamaziere A, Richard D, Barbe U, Kefi K, Bausero P, Wolf $\mathrm{C}$ et al. Differential distribution of DHA-phospholipids in rat brain after feeding: A lipidomic approach. Prostaglandins Leukot Essent Fatty Acids 2011; 84: 7-11. 
36 Little SJ, Lynch MA, Manku M, Nicolaou A. Docosahexaenoic acid-induced changes in phospholipids in cortex of young and aged rats: a lipidomic analysis. Prostaglandins Leukot Essent Fatty Acids 2007; 77: 155-162.

37 Rao JS, Ertley RN, DeMar JC Jr., Rapoport SI, Bazinet RP, Lee HJ. Dietary n-3 PUFA deprivation alters expression of enzymes of the arachidonic and docosahexaenoic acid cascades in rat frontal cortex. Mol Psychiatry 2007; 12: 151-157.

38 Strokin M, Sergeeva M, Reiser G. Docosahexaenoic acid and arachidonic acid release in rat brain astrocytes is mediated by two separate isoforms of phospholipase A2 and is differently regulated by cyclic AMP and $\mathrm{Ca} 2+$. Br J Pharmacol 2003; 139: 1014-1022.

39 Tiemeier H, van Tuijl HR, Hofman A, Kiliaan AJ, Breteler MM. Plasma fatty acid composition and depression are associated in the elderly: the Rotterdam Study. Am J Clin Nutr 2003; 78: 40-46.

40 Ricart-Jane D, Rodriguez-Sureda V, Benavides A, Peinado-Onsurbe J, Lopez-Tejero MD, Llobera M. Immobilization stress alters intermediate metabolism and circulating lipoproteins in the rat. Metabolism 2002; 51: 925-931.

41 Chuang JC, Cui H, Mason BL, Mahgoub M, Bookout AL, Yu HG et al. Chronic socialdefeat stress disrupts regulation of lipid synthesis. J Lipid Res 2010; 51: 1344-1353.

42 Giovacchini G, Chang MC, Channing MA, Toczek M, Mason A, Bokde AL et al. Brain incorporation of [11C]arachidonic acid in young healthy humans measured with positron emission tomography. J Cereb Blood Flow Metab 2002; 22: 1453-1462.

43 Riederer M, Ojala PJ, Hrzenjak A, Graier WF, Malli R, Tritscher M et al. Acyl chaindependent effect of lysophosphatidylcholine on endothelial prostacyclin production. J Lipid Res 2010; 51: 2957-2966.

44 Kim WJ, Lee MJ, Park MA, Jung JS, Uhlinger DJ, Kwak JY. Dexamethasone enhances phospholipase D activity in M-1 cells. Exp Molecular Med 2000; 32: 170-177.
45 Kerr DS, Campbell LW, Thibault O, Landfield PW. Hippocampal glucocorticoid receptor activation enhances voltage-dependent $\mathrm{Ca} 2+$ conductances: relevance to brain aging. Proc Natl Acad Sci U S A 1992; 89: 8527-8531.

$46 \mathrm{Kim} \mathrm{JH}$, Lee BD, Kim Y, Lee SD, Suh PG, Ryu SH. Cytosolic phospholipase A2-mediated regulation of phospholipase D2 in leukocyte cell lines. J Immunol 1999; 163: 5462-5470.

47 Oliveira TG, Di Paolo G. Phospholipase D in brain function and Alzheimer's disease. Biochim Biophys Acta 2010; 1801: 799-805.

48 Oliveira TG, Chan RB, Tian H, Laredo M, Shui G, Staniszewski A et al. Phospholipase d2 ablation ameliorates Alzheimer's disease-linked synaptic dysfunction and cognitive deficits. J Neurosci 2010; 30: 16419-16428.

49 Feng PF, Huang CF. Phospholipase D-mTOR signaling is compromised in a rat model of depression. J Psychiatr Res 2013; 47: 579-585.

50 Song C, Zhang XY, Manku M. Increased phospholipase a2 activity and inflammatory response but decreased nerve growth factor expression in the olfactory bulbectomized rat model of depression: effects of chronic ethyl-eicosapentaenoate treatment. J Neurosci 2009; 29: 14-22.

51 Tatebayashi Y, Nihonmatsu-Kikuchi N, Hayashi Y, Yu X, Soma M, Ikeda K. Abnormal fatty acid composition in the frontopolar cortex of patients with affective disorders. Transl Psychiatry 2012; 2: e204.

52 Schwarz E, Prabakaran S, Whitfield P, Major H, Leweke FM, Koethe D et al. High throughput lipidomic profiling of schizophrenia and bipolar disorder brain tissue reveals alterations of free fatty acids, phosphatidylcholines, and ceramides. J Proteome Res 2008; 7: 4266-4277.

53 Taha AY, Cheon Y, Ma K, Rapoport SI, Rao JS. Altered fatty acid concentrations in prefrontal cortex of schizophrenic patients. J Psychiatr Res 2013; 47: 636-643.

54 Rao JS, Kim HW, Harry GJ, Rapoport SI, Reese EA. Increased neuroinflammatory and arachidonic acid cascade markers, and reduced synaptic proteins, in the postmortem frontal cortex from schizophrenia patients. Schizophr Res 2013; 147: 24-31.

Supplementary Information accompanies the paper on the Molecular Psychiatry website (http://www.nature.com/mp) 